\title{
A SURVEY ON LOCAL COHOMOLOGY AND D-MODULES
}

\author{
SANTIAGO ZARZUELA \\ Departament d'Àlgebra i Geometria, Universitat de Barcelona \\ Gran Via 585, E-08007 Barcelona, Spain \\ E-mail: zarzuela@mat.ub.es
}

1. Introduction. Local Cohomology, in Commutative Algebra, may be seen as the algebraic counterpart to the cohomology groups of a topological space $X$ with coefficients in an Abelian sheaf on $X$, and supports in a locally closed subspace, introduced by A. Grothendieck.

It may be defined as follows: Let $R$ be a (commutative) noetherian ring, $I \subset R$ an ideal of $R$, and $M$ an $R$-module. The local cohomology modules of $M$ supported on $I$ are the right derived functors of the functor of sections of $M$ with support on $I$ :

$$
H_{I}^{r}(M)=\mathrm{R}^{r}\left(\Gamma_{I}(M)\right)
$$

where $\Gamma_{I}(M)=\bigcup_{n \geq 0}\left(0: I^{n} M\right)$. It may be seen that:

$$
H_{I}^{r}(M) \simeq \operatorname{ind} . \lim \operatorname{Ext}_{R}^{r}\left(R / I^{n}, M\right) \simeq H^{r}\left(C\left(a_{1}, \ldots, a_{t} ; M\right)\right)
$$

where $C\left(a_{1}, \ldots, a_{t} ; M\right)$ denotes the Čech complex of $M$ with respect to any system of generators $a_{1}, \ldots, a_{t}$ of $I$, with

$$
C\left(a_{1}, \ldots, a_{t} ; M\right)_{r}=\bigoplus_{i_{1}<\cdots<i_{r}} M_{a_{i_{1}} \cdots a_{i_{r}}}
$$

and differentials given by the natural localization morphisms.

After R. Hartshorne's notes on A. Grothendieck's 1961 Harvard University Seminar [14] and subsequent work [15], Local Cohomology became a fundamental and effective tool in Commutative Algebra. It provides a great amount of information about the module $M$ and the ideal $I$, mainly when $M$ is a finitely generated $R$-module. Let us recall some basic vanishing (and non-vanishing) results:

a) Assume that $M$ is a finitely generated $R$-module. Then, for any $I$

$$
\operatorname{grade}_{I}(M)=\min \left\{r \mid H_{I}^{r}(M) \neq 0\right\}<\infty .
$$

2000 Mathematics Subject Classification: Primary 13D45; Secondary 52C35.

Partially supported by the DGCYT PB97-0893.

The paper is in final form and no version of it will be published elsewhere. 
b) For any $I$ and $M$

$$
H_{I}^{r}(M)=0, \text { for any } r>\operatorname{dim}_{R}(M) .
$$

c) If $(R, \mathfrak{m})$ is a local ring with maximal ideal $\mathfrak{m}$, and $M$ is a finitely generated $R$ module then

$$
\operatorname{dim}_{R}(M)=\max \left\{r \mid H_{\mathfrak{m}}^{r}(M) \neq 0\right\}
$$

For any ideal $I \subset R$ let the arithmetical rank $\operatorname{ara}(I)$ of $I$ be the least number of elements of $R$ required to generate an ideal which has the same radical as $I$, that is, the minimal number of elements needed to define set theoretically the same variety as $I$. Then

d) $H_{I}^{r}(R)=0$ for any $r>\operatorname{ara}(I)$.

Usually it is difficult to determine the structure (vanishing, support, injective dimension, ...) of the local cohomology modules as $R$-modules, mainly due to the fact that, even for the ring $R$ itself, they are in general not finitely generated.

In this sense, several deep conjectures concerning local cohomology modules were stated by R. Hartshorne, a central idea behind them being that local cohomology modules behave as finitely generated ones in some aspects, and this would be enough to obtain useful information about their structure, see C. Huneke [16] for an account of these conjectures.

Let us mention now the following result proved a few years ago:

THEOREM 1 (1993). Let $R$ be a regular ring containing a field and $I \subset R$ an ideal of $R$. Let $H_{I}^{r}(R)$ be the $r$-th local cohomology module of $R$ supported on $I$. Then the following hold:

i) $H_{\mathfrak{m}}^{j}\left(H_{I}^{r}(R)\right)$ is injective for any maximal ideal $\mathfrak{m}$ of $R$.

ii) inj.dim ${ }_{R}\left(H_{I}^{r}(R)\right) \leq \operatorname{dim}_{R} H_{I}^{r}(R)$.

iii) The set of associated primes of $H_{I}^{r}(R)$ is finite.

iv) The Bass numbers of $H_{I}^{r}(R)$ are finite.

(Recall that if $M$ is an $R$-module and $\mathfrak{p}$ is a prime ideal of $R$, the Bass number $\mu_{R}^{i}(\mathfrak{p}, M)$ is the number of copies of the injective envelope $E_{R}(R / \mathfrak{p})$ of $R / \mathfrak{p}$ that appear in the $i$-th step of a minimal injective resolution of $M$, and it is given by

$$
\mu_{R}^{i}(\mathfrak{p}, M)=\operatorname{dim}_{k(\mathfrak{p})} \operatorname{Ext}_{R_{\mathfrak{p}}}^{i}\left(k(\mathfrak{p}), M_{\mathfrak{p}}\right) .
$$

The proof of this theorem depends on the characteristic of the field, and was obtained by:

- C. Huneke and R.Y. Sharp [17] if the field is of positive characteristic, by using the Frobenius.

- G. Lyubeznik [18] if the field is of characteristic zero, by using the theory of $\mathcal{D}$ modules: When $R$ is a polynomial ring or a formal power series ring over a field of characteristic zero, the local cohomology modules of $R$ have a $\mathcal{D}$-module structure, where $\mathcal{D}$ is the ring of differential operators. In fact, they are holonomic as $\mathcal{D}$ modules. 
The main goal of this paper is to survey some recent results by J. Àlvarez [1], [2] and J. Àlvarez, R. García and S. Zarzuela [3] on the structure of the local cohomology modules of a polynomial ring $R$ over a field $k$ of characteristic zero, particularly when the support is a monomial ideal or, more generally, the defining ideal of an arrangement of linear varieties in the affine space $\mathbb{A}_{k}^{n}$. The obtained information is essentially given by their structure as $\mathcal{D}$-modules, specially by their characteristic cycles.

Namely, if $I \subset R$ is a square free monomial ideal, we will see how the multiplicities of the characteristic cycles of the local cohomology modules $H_{I}^{r}(R)$ recover the basic combinatorical invariants of the simplicial complex whose Stanley-Reisner ring is $R / I$. And if $I \subset R$ is the defining ideal of an arrangement of linear varieties on the affine space $\mathbb{A}_{k}^{n}$ we shall compute the multiplicities of the characteristic cycles of the corresponding local cohomology modules as the Betti numbers of the complement of the arrangement defined by $I$.

No proofs will be given here. We will use M. Brodmann and R. Y. Sharp [8] as a basic reference for Local Cohomology, and H. Matsumura [19], W. Bruns and J. Herzog [9] for any unexplained terminology of Commutative Algebra. Finally, all the concepts we need on $\mathcal{D}$-module theory can be found in S. C. Coutinho [10], J.-E. Björk [5] and A. Borel et al. [7].

This paper is based on the talk given by the author at the Workshop on Differential Galois Theory, held at the Mathematical Research and Conference Center of the Institute of Mathematics of the Polish Academy of Sciences, in Będlewo, from May 28th to June 1st 2001. The author would like to thank the organizers, Teresa Crespo and Zbigniew Hajto, for their work and for inviting him to participate in it. Thanks are also due to Josep Àlvarez and Ricardo García for their valuable comments improving the readability of this paper.

2. Holonomic $\mathcal{D}$-modules, local cohomology, and characteristic cycles. In this section we will recall some basic concepts on $\mathcal{D}$-module theory in order to fix the aspects we are interested in to study local cohomology modules.

Let $R=k\left[x_{1}, \ldots, x_{n}\right]$ be the polynomial ring in $n$ variables over a field $k$ of characteristic zero. Recall that in this case the ring of differential operators of $R$ is isomorphic to the (noncommutative) $R$-algebra

$$
\mathcal{D}=R<\partial_{1}, \ldots, \partial_{n}>
$$

generated by the partial derivatives $\partial_{i}=\frac{d}{d x_{i}}$ with the relations given by

$$
\partial_{i} \partial_{j}=\partial_{j} \partial_{i}, \quad \partial_{i} r-r \partial_{i}=\frac{d r}{d x_{i}}
$$

where $r \in R$. This algebra, usually called the Weyl algebra of $R$, is left and right Noetherian. Here we will always consider left $\mathcal{D}$-modules.

The category of holonomic $\mathcal{D}$-modules is a full abelian subcategory of the category of $\mathcal{D}$-modules with very good properties. In particular, holonomic $\mathcal{D}$-modules are of finite length as $\mathcal{D}$-modules. As the basic examples for our purposes we have:

- $R$ itself is holonomic as a $\mathcal{D}$-module. 
- All the localizations $R_{f}, f \in R$, are holonomic.

- From the construction of the Čech complex we have that for any ideal $I \subset R$, the local cohomology modules $H_{I}^{r}(R)$ are holonomic $\mathcal{D}$-modules too.

The algebra $\mathcal{D}$ has an increasing filtration $\left\{\Sigma_{v}\right\}_{v \geq 0}$ (the order filtration) such that its associated graded ring is isomorphic to a (commutative) polynomial ring in $n$ variables over $R$. Namely,

$$
g r_{\Sigma}(\mathcal{D})=\bigoplus_{v \geq-1} \Sigma_{v+1} / \Sigma_{v}=R\left[y_{1}, \ldots, y_{n}\right]
$$

where $y_{i}=\bar{\partial}_{i} \in \Sigma_{1} / \Sigma_{0}$ and $\Sigma_{-1}=0$.

Also, for any finitely generated $\mathcal{D}$-module $M$ there exists a good filtration, that is, there is an increasing sequence of finitely generated $R$-submodules $\Gamma_{0} \subseteq \Gamma_{1} \subseteq \cdots \subseteq M$ satisfying:

i) $\bigcup \Gamma_{k}=M$

ii) $\Sigma_{v} \Gamma_{k} \subseteq \Gamma_{v+k}, \forall k, v \geq 0$,

and such that $g r_{\Gamma}(M)=\bigoplus_{k \geq-1} \Gamma_{k+1} / \Gamma_{k} \quad\left(\Gamma_{-1}=0\right)$ is a finitely generated $g r_{\Sigma}(\mathcal{D})$ module.

By Bernstein's inequality we have that $n \leq \operatorname{dim} \operatorname{gr}_{\Gamma}(M) \leq 2 n$, the holonomic modules being exactly those for which $n=\operatorname{dim} g r_{\Gamma}(M)$.

Now, for any finitely generated $\mathcal{D}$-module $M$ we can consider its characteristic ideal $J(M)$ :

$$
J(M)=\sqrt{A n n_{g r_{\Sigma}(\mathcal{D})}\left(g r_{\Gamma}(M)\right)},
$$

which is independent of the chosen good filtration, as well as its characteristic variety $C(M)$ :

$$
C(M)=V(J(M)) \subseteq \operatorname{Spec}\left(g r_{\Sigma}(\mathcal{D})\right) .
$$

Observe that if $M$ is holonomic then $\operatorname{dim} C(M)=n$.

The characteristic variety allows to describe the support of $M$ as $R$-module, since if

$$
\pi: \operatorname{Spec}\left(R\left[y_{1}, \ldots, y_{n}\right]\right) \rightarrow \operatorname{Spec}(R)
$$

is the natural contraction then $\operatorname{Supp}_{R}(M)=\pi(C(M))$.

The characteristic cycle of $M$ is then defined as:

$$
C C(M)=\sum m_{\alpha} V_{\alpha}
$$

where the sum is taken over all the irreducible components $V_{\alpha}$ of $C(M)$, and the $m_{\alpha}$ 's are certain attached multiplicities, in such a way that

$$
V_{\alpha} \subset C(M) \Leftrightarrow m_{\alpha} \neq 0 .
$$

The characteristic cycle is additive with respect to exact sequences.

Local cohomology modules are regular: A holonomic $\mathcal{D}$-module is called regular if there exists a good filtration such that the annihilator of the associated graded module is a radical ideal.

Over $\mathbb{C}$, the field of complex numbers, the characteristic variety of a holonomic $\mathcal{D}$ module $M$ has a particular shape (see [21]). Namely, if we think of $\operatorname{Spec}\left(g r_{\Sigma}(\mathcal{D})\right)$ as $T^{*} X$, 
the cotangent bundle of $X=\operatorname{Spec}(R)$, and then consider the projection

$$
\pi: T^{*} X \rightarrow X
$$

one can see that the irreducible components $V_{\alpha}$ of $C(M)$ are of the form

$$
V_{\alpha}=T_{Y_{\alpha}}^{*} X
$$

where $Y_{\alpha}$ is the projection of the component and $T_{Y_{\alpha}}^{*} X$ the conormal bundle of $X$ with respect to $Y_{\alpha}$.

As a consequence, the characteristic variety of a holonomic module $M$ has a decomposition of the form

$$
C(M)=\bigcup T_{Y_{\alpha}}^{*} X
$$

where $Y_{\alpha}$ are the projections of the irreducible components of $C(M)$. In particular, we have a description of the support of $M$ as an $R$-module. Namely,

$$
\operatorname{Supp}_{R} M=\bigcup Y_{\alpha} \text {. }
$$

By flat base change, and over any field $k$ of characteristic zero, one has a similar description of the characteristic cycles of the local cohomology modules $H_{I}^{r}(R)$, and so an explicit description of their support. In particular, one can determine from their characteristic cycles when they vanish. Furthermore:

Theorem 2 (J. Àlvarez [1]). For any ideal $I \subset R$, and for any $r \geq 0$, the multiplicities of the characteristic cycle of $H_{I}^{r}(R)$ are invariants of $R / I$.

For any $I \subset R$ set

$$
C C\left(H_{I}^{n-i}(R)\right)=\sum m_{\alpha} T_{Y_{\alpha}}^{*} X
$$

We may then consider the following numbers:

$$
\gamma_{p, i}(R / I):=\left\{\sum m_{\alpha}: \operatorname{dim} Y_{\alpha}=p\right\}
$$

These are also invariants of $R / I$, and if $\operatorname{dim} R / I=d$ they satisfy the following nice properties:

Proposition 3 (J. Àlvarez [1]). With the above notations,

i) $\gamma_{p, i}(R / I)=0$ if $i>d$.

ii) $\gamma_{p, i}(R / I)=0$ if $p>i$.

iii) $\gamma_{d, d}(R / I) \neq 0$.

In particular, we can consider the triangular matrix given by the invariants $\gamma_{p, i}$ :

$$
\left(\begin{array}{ccc}
\gamma_{0,0} & \cdots & \gamma_{0, d} \\
& \ddots & \vdots \\
& & \gamma_{d, d}
\end{array}\right)
$$

that we call the characteristic matrix of $I$.

Now our objective is twofold:

1) To compute the multiplicities of local cohomology modules.

2) To give adequate interpretations of these multiplicites. 
As we shall see, one is able to do this for local cohomology modules supported on monomial ideals and, more generally, for the definition ideals of arrangements of linear varieties.

3. Local cohomology supported on monomial ideals. The study of local cohomology modules supported on monomial ideals has received much attention recently, and they have been explicitely computed from several points of view and using different techniques, see for instance F. Barkats [4], U. Walther [24], M. Mustaţă [20], and K. Yanagawa [25].

In this section we want to review a formula by J. Àlvarez [1], describing the characteristic cycles of the local cohomology modules supported on a monomial ideal by means of the face ideals in its minimal primary decomposition. Also, we will see how the corresponding attached multiplicities provide combinatorial information.

The easiest case is when $I$ is a face ideal: Let $I=\mathfrak{p}=\left(x_{i_{1}}, \ldots, x_{i_{h}}\right) \subset R, h=\operatorname{ht}(\mathfrak{p})$. Then

$$
H_{\mathfrak{p}}^{r}(R)=0 \text { if } r \neq h \quad \text { and } \quad C C\left(H_{\mathfrak{p}}^{h}(R)\right)=T_{\left\{x_{i_{1}}=\cdots=x_{i_{h}}=0\right\}}^{*} X .
$$

For the general case, and since it is enough to compute local cohomology for radical ideals, we may always assume that $I$ is a squarefree monomial ideal.

So let $I=\mathfrak{p}_{1} \cap \ldots \cap \mathfrak{p}_{m}$ be a minimal primary decomposition, where $\mathfrak{p}_{j}$ are face ideals, $j=1, \ldots, m$. Note that sums $\mathfrak{p}_{j_{1}}+\cdots+\mathfrak{p}_{j_{k}}$ of face ideals are again face ideals. Then,

TheOrem 4 (J. Àlvarez [1]). With the above notations,

$$
\begin{aligned}
C C\left(H_{I}^{n-i}(R)\right)= & \sum_{\mathfrak{p}_{j} \in \Omega_{1, i}} C C\left(H_{\mathfrak{p}_{j}}^{n-i}(R)\right)+\sum_{\mathfrak{p}_{j}+\mathfrak{p}_{k} \in \Omega_{2, i}} C C\left(H_{\mathfrak{p}_{j}+\mathfrak{p}_{k}}^{n-i+1}(R)\right) \\
& +\cdots+\sum_{\mathfrak{p}_{1}+\cdots+\mathfrak{p}_{m} \in \Omega_{m, i}} C C\left(H_{\mathfrak{p}_{1}+\cdots+\mathfrak{p}_{m}}^{n-i+(m-1)}(R)\right)
\end{aligned}
$$

where $\Omega_{j, i}$ are subsets of the sets of sums of $j$ face ideals that one computes by using an algorithm. They have height $n-i+j-1$.

The algorithm is based on a recursive use of Mayer-Vietoris sequences, by applying the additivity of the characteristic cycle with respect to short exact sequences.

One can extract some consequences from this formula. For instance, the face ideals in the sets $\Omega_{j, i}$ describe the support of the local cohomology modules $H_{I}^{n-i}(R)$. From the algorithm one may also see that for any $i$,

$$
\Omega_{1, i}=\left\{\mathfrak{p}_{j} \mid \text { ht }\left(\mathfrak{p}_{j}\right)=n-i\right\} .
$$

In particular, if $\mathfrak{p}_{j}$ is a face ideal in the primary decomposition of $I$,

$$
\mathfrak{p}_{j} \in \operatorname{Supp}_{R}\left(H_{I}^{n-i}(R)\right) \Leftrightarrow \operatorname{ht}\left(\mathfrak{p}_{j}\right)=n-i,
$$

and in this case it is a minimal prime ideal of $H_{I}^{n-i}(R)$. (But there can be other minimal primes.)

From the theorem we also have that

$$
\gamma_{p, i}(R / I)=\# \Omega_{i-p+1, i} .
$$

And in terms of these invariants one may: 
- Give a vanishing criterion for $H_{I}^{n-i}(R)$ :

$$
H_{I}^{n-i}(R)=0 \Leftrightarrow \gamma_{p, i}=0 \forall p .
$$

- Compute the cohomological dimension $\operatorname{cd}(I, R)$ of $I$, that is, $\max \left\{r \mid H_{I}^{r}(R) \neq 0\right\}$ :

$$
\operatorname{cd}(R, I)=n-\min \left\{i \mid \gamma_{p, i} \neq 0 \text { for some } p\right\} .
$$

- Give a Cohen-Macaulayness criterion for $R / I$ :

$$
R / I \text { is Cohen-Macaulay } \Leftrightarrow \gamma_{p, i}=0 \forall i \neq \operatorname{dim} R / I, \forall p .
$$

Now, let $\Delta$ be a simplicial complex on the set of vertices $\left\{x_{1}, \ldots, x_{n}\right\}$ and

$$
I_{\Delta}=\left(x_{1}^{\alpha_{1}} \cdots x_{n}^{\alpha_{n}} \mid\left(\alpha_{1}, \ldots, \alpha_{n}\right) \in\{0,1\}^{n},\left\{x_{i}^{\alpha_{i}} \mid \alpha_{1}=1\right\} \notin \Delta\right\}
$$

the defining ideal of the Stanley-Reisner ring of $\Delta$ :

$$
k[\Delta]:=k\left[x_{1}, \ldots, x_{n}\right] / I_{\Delta} .
$$

$I_{\Delta}$ is a square free monomial ideal, and in this way there is a bijection between the set of simplicial complexes on the set of vertices $\left\{x_{1}, \ldots, x_{n}\right\}$ and the set of square free monomial ideals in $R$. See R. Stanley [22] for more details.

Observe that sums of face ideals in the minimal primary decomposition of $I_{\Delta}$ correspond to the intersection of maximal faces of $\Delta$.

Let $\operatorname{dim} \Delta=\operatorname{dim} k[\Delta]-1=d-1$, and consider the $f$-vector of $\Delta$ :

$$
f(\Delta)=\left(f_{-1}, \ldots, f_{d-1}\right),
$$

where $f_{k}$ is the number of $k$-dimensional faces of $\Delta$, and the $h$-vector of $\Delta$ :

$$
h(\Delta)=\left(h_{0}, h_{1}, \ldots, h_{d}\right) .
$$

Both are related by means of the equality

$$
\sum_{i=0}^{d} f_{i-1}(t-1)^{d-i}=\sum_{i=0}^{d} h_{i} t^{i} .
$$

Finally, consider the characteristic matrix of $I_{\Delta}$ :

$$
\left(\begin{array}{ccc}
\gamma_{0,0} & \cdots & \gamma_{0, d} \\
& \ddots & \vdots \\
& & \gamma_{d, d}
\end{array}\right),
$$

and denote

$$
\mathcal{B}_{k}:=\gamma_{k, k}-\gamma_{k, k+1}+\cdots+(-1)^{d-k} \gamma_{k, d} .
$$

Then we have the following relations:

Proposition 5 (J. Àlvarez [1]). With the above notations,

i) $f_{k}=\left(\begin{array}{c}d \\ k+1\end{array}\right) \mathcal{B}_{d}+\left(\begin{array}{c}d-1 \\ k+1\end{array}\right) \mathcal{B}_{d-1}+\cdots+\left(\begin{array}{c}k+1 \\ k+1\end{array}\right) \mathcal{B}_{k+1}$.

ii) $1=f_{-1}=\mathcal{B}_{d}+\cdots+\mathcal{B}_{0}=\sum(-1)^{p+i} \gamma_{p, i}$.

iii) The Euler characteristic of $\Delta$ is $\chi(\Delta)=1-\mathcal{B}_{0}$.

iv) $h_{k}=(-1)^{k}\left(\left(\begin{array}{c}d \\ k\end{array}\right) \mathcal{B}_{0}+\left(\begin{array}{c}d-1 \\ k\end{array}\right) \mathcal{B}_{1}+\cdots+\left(\begin{array}{c}k \\ k\end{array}\right) \mathcal{B}_{d-k}\right)$. 
Here we would like to point out that the invariants $\gamma_{p, i}$ are finer than the $f$-vector or the $h$-vector, but they are equivalent if the ring $R / I_{\Delta}$ is Cohen-Macaulay.

Example. Let $R=k\left[x_{1}, x_{2}, x_{3}, x_{4}, x_{5}\right]$. Consider:

$$
I_{\Delta_{1}}=\left(x_{1}, x_{3}\right) \cap\left(x_{1}, x_{4}\right) \cap\left(x_{1}, x_{5}\right) \cap\left(x_{2}, x_{3}\right) \cap\left(x_{2}, x_{4}\right) .
$$

Then $f\left(\Delta_{1}\right)=(1,5,9,5)$ and the characteristic matrix of $I_{\Delta_{1}}$ is

$$
\left(\begin{array}{llll}
0 & 0 & 0 & 0 \\
& 0 & 0 & 2 \\
& & 0 & 6 \\
& & & 5
\end{array}\right) .
$$

Now, consider

$$
I_{\Delta_{2}}=\left(x_{1}, x_{4}\right) \cap\left(x_{1}, x_{5}\right) \cap\left(x_{2}, x_{5}\right) \cap\left(x_{3}, x_{5}\right) \cap\left(x_{4}, x_{5}\right) \cap\left(x_{1}, x_{2}, x_{3}\right) .
$$

Then, as before, $f\left(\Delta_{2}\right)=(1,5,9,5)$ while the characteristic matrix of $I_{\Delta_{2}}$ is

$$
\left(\begin{array}{llll}
0 & 0 & 1 & 1 \\
& 0 & 2 & 4 \\
& & 1 & 7 \\
& & & 5
\end{array}\right) .
$$

There is still another combinatorial meaning for the multiplicities of the characteristic cycles of the local cohomology modules supported on monomial ideals:

The ring $R=k\left[x_{1}, \ldots, x_{n}\right]$ has a natural $\mathbb{Z}^{n}$-graduation given by $\operatorname{deg}\left(x_{i}\right)=\epsilon_{i}$ where $\epsilon_{1}, \ldots, \epsilon_{n}$ is the canonical basis of $\mathbb{Z}^{n}$. There is also an obvious bijection between the degrees $\alpha \in\{0,1\}^{n}$ and the face ideals of the form $\mathfrak{p}_{\alpha}=<x_{i}>_{\alpha_{i}=1}$. Note that if $\mathfrak{p}_{\alpha}$ is a face ideal then $|\alpha|=$ ht $\left(\mathfrak{p}_{\alpha}\right)$, and that monomial ideals are exactly the homogeneous ones for this $\mathbb{Z}^{n}$ graduation of $R$.

On the other hand, for any given $\mathbb{Z}^{n}$-graded $R$-module $M$ we may consider a minimal $\mathbb{Z}^{n}$-graded free resolution

$$
L_{\bullet}(M): 0 \rightarrow L_{r} \rightarrow L_{r-1} \rightarrow \cdots \rightarrow L_{0} \rightarrow M \rightarrow O
$$

where

$$
L_{j}=\oplus_{\alpha \in \mathbb{Z}^{n}} R(-\alpha)^{\beta j, \alpha}
$$

and $\beta_{j, \alpha}$ are the graded Betti numbers. The Castelnuovo-Mumford regularity of $M$ denoted $\operatorname{reg}(M)$ is then defined as

$$
\operatorname{reg}(M):=\max \left\{|\alpha|-j \mid \beta_{j, \alpha} \neq 0\right\} .
$$

For a given integer $i$, the $i$-linear strand of $L_{\bullet}(M)$ is the subcomplex

$$
L_{\bullet}^{<i>}(M): 0 \rightarrow L_{r}^{<i>} \rightarrow L_{r-1}^{<i>} \rightarrow \cdots \rightarrow L_{0}^{<i>} \rightarrow O
$$

where

$$
L_{j}^{<i>}=\oplus_{|\alpha|=j+i} R(-\alpha)^{\beta j, \alpha} .
$$

Now, recall that for a given square free monomial ideal $I \subset R$, the Alexander dual of $I$ is the ideal 


$$
I^{\vee}=<\prod_{i \in S} x_{i} \mid S \subset\{1, \ldots, n\}, \prod_{i \in S^{c}} x_{i} \notin I>
$$

In fact, the simplicial complex associated to the Stanley-Reisner ring $R / I^{\vee}$ is the Alexander dual of the simplicial complex associated to $R / I$, see [22].

Let $\beta_{j, \alpha}$ be the graded Betti numbers of the Alexander dual of $I$. Then:

Theorem 6 (J. Àlvarez [2], J. Àlvarez, R. García, and S. Zarzuela [3]). For any $\alpha$ $\notin\{0,1\}^{n}$, the Betti number $\beta_{j, \alpha}=0$. Furthermore, if $\mathfrak{p}_{\alpha} \in R$ is a face ideal, $X_{\alpha}$ is the linear variety defined by $\mathfrak{p}_{\alpha}$, and $m_{i, \alpha}$ the multiplicity of $T_{X_{\alpha}}^{*} X$ in

$$
C C\left(H_{I}^{n-i}(R)\right)=\sum m_{i, \alpha} T_{X_{\alpha}}^{*} X,
$$

then

i) $\beta_{j, \alpha}=0$ if $\mathfrak{p}_{\alpha} \notin \Omega_{j, i}$ for any $i$.

ii) If $\mathfrak{p}_{\alpha} \in \Omega_{j, i}$ then

$$
\beta_{j, \alpha}=m_{i, \alpha}, \text { with } i=n-|\alpha|-j+1 \text {. }
$$

Note that each local cohomology module $H_{I}^{n-i}(R)$ provides exactly the Betti numbers of the $n-i-1$ linear strand in the minimal free resolution of $I^{\vee}$. As a consequence we get the following result, first proved by J. A. Eagon and V. Reiner [11]:

- $I$ is Cohen-Macaulay if and only if $I^{\vee}$ has a linear resolution.

As well as the following generalization by N. Terai [23]:

- $\operatorname{reg}\left(I^{\vee}\right)=\operatorname{cd}(R, I)=$ proj.dim $(R / I)$.

Two different proofs of the above theorem may be given:

i) One may see that the local cohomology modules $H_{I}^{n-i}(R)$ are also $\mathbb{Z}^{n}$-graded, and that the multiplicities of their characteristic cycles are equal to the lengths of appropriate graded pieces. Then one uses a result by M. Mustaţă [20] that identifies these lengths as the Betti numbers of the Alexander dual of $I$ [3].

ii) Consider the Taylor resolution of the Alexander dual of $I$. Then one sees that the algorithm used to compute the characteristic cycles of the local cohomology modules $H_{I}^{n-i}(R)$ corresponds to an algorithm to get a minimal free resolution for the Alexander dual of $I$ from its Taylor resolution [2].

4. Local cohomology and arrangements of linear varieties. Let $I$ be the definition ideal of an arrangement of linear varieties in the affine space $\mathbb{A}_{k}^{n}$. In this section we want to relate, when $k$ is the field of real or complex numbers, the multiplicities of the characteristic cycles of the local cohomology modules supported on $I$ with the Betti numbers of the complement of the arrangement in the afine space. This will provide a purely algebraic interpretation of these Betti numbers.

Let $k$ be a field and $R=k\left[x_{1}, \ldots, x_{n}\right]$. Consider an arrangement of linear varieties $X$ in the affine space $\mathbb{A}_{k}^{n}$ defined by the ideal $I=\mathfrak{p}_{\alpha_{1}} \cap \ldots \cap \mathfrak{p}_{\alpha_{m}} \subseteq R$.

The arrangement $X$ defines a partially ordered set $P(X)$ whose elements correspond to the intersections of irreducible components of $X$. Observe that $P(X)$ is the poset 
associated to the subvarieties

$$
X_{\alpha}=V\left(\mathfrak{p}_{\alpha}\right), \text { where } \mathfrak{p}_{\alpha}=\mathfrak{p}_{\alpha_{i_{1}}}+\cdots+\mathfrak{p}_{\alpha_{i_{k}}}
$$

ordered by reverse inclusion.

For any $p \in P(X)$, denote by $X_{p}$ the linear affine variety corresponding to $p$, by $I_{p}$ the radical ideal which defines $X_{p}$, and by $h(p)$ the codimension of $X_{p}$, that is, the height of the ideal $I_{p}$.

Further for any $p \in P(X)$, let $K(>p)(K(\geq p))$ be the simplicial complex attached to the subposet

$$
\begin{gathered}
\{q \in P(X) \mid q>p\} \\
(\{q \in P(X) \mid q \geq p\})
\end{gathered}
$$

of $P(X)$.

For $k=\mathbb{R}$, the field of real numbers, M. Goresky and R. MacPherson gave a formula for the homology of the complement of $X$ in $\mathbb{A}_{k}^{n}$ that may be stated as follows:

Theorem 7 (M. Goresky and R. MacPherson [13]). With the above notations,

$$
\tilde{H}_{i}\left(\mathbb{A}_{\mathbb{R}}^{n}-X ; \mathbb{Z}\right) \simeq \bigoplus_{p \in P(X)} H^{h(p)-i-1}(K(\geq p), K(>p) ; \mathbb{Z})
$$

In particular, this formula says that the Betti numbers of the complement of $X$ can be computed as the sum of non-negative integers, one for each non-empty intersection of irreducible components of $X$. These integers are, in fact, dimensions of certain Morse groups [13]. Other versions of this formula have also been proven by several authors. In particular, A. Björner and T. Ekedahl [6] have proved a $l$-adic version of it.

Now, we can give a description of these Betti numbers, for $k=\mathbb{R}$ or $k=\mathbb{C}$, in terms of the multiplicities of the characteristic cycles of the local cohomology modules $H_{I}^{r}(R)$. Namely,

Theorem 8 (J. Àlvarez, R. García, S. Zarzuela [3]). Let $k$ be a field of characteristic zero. With the above notations,

i) $C C\left(H_{I}^{r}(R)\right)=\sum_{p \in P(X)} m_{r, p} T_{X_{p}}^{*} \mathbb{A}_{k}^{n}$.

ii) If $k=\mathbb{R}$ is the field of real numbers, then

$$
\operatorname{dim}_{\mathbb{Q}} \tilde{H}_{i}\left(\mathbb{A}_{\mathbb{R}}^{n}-X ; \mathbb{Q}\right)=\sum_{p \in P(X)} m_{i+1, p}
$$

iii) If $k=\mathbb{C}$ is the field of complex numbers, then

$$
\operatorname{dim}_{\mathbb{Q}} \tilde{H}_{i}\left(\mathbb{A}_{\mathbb{C}}^{n}-X ; \mathbb{Q}\right)=\sum_{p \in P(X)} m_{i+1-h(p), p} .
$$

(The formula for the case $k=\mathbb{C}$ may be obtained from the case $k=\mathbb{R}$ regarding a complex arrangement in $\mathbb{A}_{\mathbb{C}}^{n}$ as a real arrangement in $\mathbb{A}_{\mathbb{R}}^{2 n}$.)

The proof of this result lies on the fact that, on the basis of the poset $P(X)$, one may construct a filtration

$$
\left\{F_{j}^{r}\right\}_{r \leq j \leq n}
$$


of the local cohomology module $H_{I}^{r}(R)$ such that

$$
F_{j}^{r} / F_{j-1}^{r} \simeq \bigoplus_{h(p)=j}\left(H_{I_{p}}^{j}(R)\right)^{\oplus m_{r, p}}
$$

where

$$
m_{r, p}=\operatorname{dim}_{k} \tilde{H}_{h(p)-r-1}(K(>p) ; k) .
$$

The additivity of the characteristic cycle with respect to short exact sequences gives then part i) in the theorem, and the formula of Goresky-MacPherson gives parts ii) and iii).

In fact, the existence of a filtration with the above properties for the local cohomology modules supported on the definition ideal of an arrangement of linear varieties is valid in any characteristic. Its proof follows closely the method used by A. Björner and T. Ekedahl [6].

Observe that, if $I$ is a monomial ideal, we may combine theorem 8 with the interpretation in terms of the Betti numbers of the minimal free resolution of the Alexander dual of $I$ obtained in theorem 6 . In this way we get an interpretation of the Betti numbers of the complement of the arrangement defined by $I$ in terms of the Betti numbers of the minimal free resolution of the Alexander dual of $I$, see also V. Gasharov, I. Peeva, and W. Welker [12].

A natural question that arises in this context is to what extent the characteristic cycles determine the local cohomology modules supported on monomial ideals. This would be the case if, for the filtrations $\left\{F_{j}^{r}\right\}_{r \leq j \leq n}$ of the local cohomology modules introduced above, all the extensions associated to the short exact sequences are trivial.

This is not the case. But, in our situation, we are able to solve all the extension problems attached to the exact short sequences, and so to precise the additional information one needs to determine the local cohomology modules [3]. But this must be done in the frame of the category of straight modules, introduced by K. Yanagawa in [25], which includes the local cohomology modules supported on monomial ideals.

\section{References}

[1] J. Àlvarez, Characteristic cycles of local cohomology modules of monomial ideals, J. Pure Appl. Algebra 150 (2000), 1-25.

[2] J. Àlvarez, Ph.D. Thesis, University of Barcelona, 2002.

[3] J. Àlvarez, R. García and S. Zarzuela, Local cohomology, arrangements of subspaces and monomial ideals, Adv. Math., to appear.

[4] F. Barkats, Calcul effectif de groupes de cohomologie locale à support dans des idéaux monomiaux, Ph.D. Thesis, Univ. Nice-Sophia Antipolis, 1995.

[5] J.-E. Björk, Rings of Differential Operators, North-Holland Math. Library, 21, NorthHolland Amsterdam, 1979.

[6] A. Björner and T. Ekedahl, Subspace arrangements over finite fields: cohomological and enumerative aspects, Adv. Math. 129 (1997), 159-187.

[7] A. Borel et al., Algebraic D-modules, Perspectives in Math. 2, Academic Press, Boston, MA, 1987. 
[8] M. P. Brodmann and R. Y. Sharp, Local Cohomology: an Algebraic Introduction with Geometric Applications, Cambridge Stud. Adv. Math. 60, Cambridge Univ. Press, Cambridge, 1998 .

[9] W. Bruns and J. Herzog, Cohen-Macaulay Rings, Cambridge Stud. Adv. Math. 39, Cambridge University Press, Cambridge, 1993.

[10] S. C. Coutinho, A Primer of Algebraic D-modules, London Math. Soc. Student Texts 33, Cambridge Univ. Press, Cambridge, 1995.

[11] J. A. Eagon and V. Reiner, Resolutions of Stanley-Reisner rings and Alexander duality, J. Pure Appl. Algebra 130 (1998), 265-275.

[12] V. Gasharov, I. Peeva, and W. Welker, Coordinate subspace arrangements and monomial ideals, preprint.

[13] M Goresky and R. MacPherson, Stratified Morse Theory, Ergeb. Math. Grenzgeb. (3) 14, Springer, Berlin, 1988.

[14] R. Hartshorne, Local Cohomology, Lecture Notes in Math. 41, Springer, Berlin, 1967.

[15] R. Hartshorne, Cohomological dimension of algebraic varieties, Ann. of Math. (2) 88 (1968), 403-450.

[16] C. Huneke, Problems on local cohomology, in: Free Resolutions in Commutative Algebra and Algebraic Geometry (Sundance, UT, 1990), 93-108, Res. Notes Math. 2, Jones and Bartlett, Boston, MA, 1992.

[17] C. Huneke and R. Y. Sharp, Bass numbers of local cohomology modules, Trans. Amer. Math. Soc. 339 (1993), 765-779.

[18] G. Lyubeznik, Finiteness properties of local cohomology modules, Invent. Math. 113 (1993), $41-55$.

[19] H. Matsumura, Commutative Ring Theory, Cambridge Stud. Adv. Math. 8, Cambridge Univ. Press, Cambridge, 1989.

[20] M. Mustaţă, Local cohomology at monomial ideals, J. Symbolic Comput. 29 (2000), 709720 .

[21] F. Pham, Singularités des systèmes différentiels de Gauss-Manin, Progr. Math. 2, Birkhäuser, Boston, MA, 1979.

[22] R. Stanley, Combinatorics and Commutative Algebra, Progr. Math. 41, Birkhäuser, Boston, MA, 1996.

[23] N. Terai, Alexander duality theorem and Stanley-Reisner rings, Sūrikaisekikenkyūsho Kōkyūroku No. 1078 (1999), 174-184 (Japanese). MR: 2001f:13033.

[24] U. Walther, Algorithmic computation of local cohomology modules and the local cohomological dimension of algebraic varieties, J. Pure Appl. Algebra 139 (1999), 303-321.

[25] K. Yanagawa, Bass numbers of local cohomology modules with supports in monomial ideals, Math. Proc. Cambridge Philos. Soc. 131 (2001), 45-60. 